
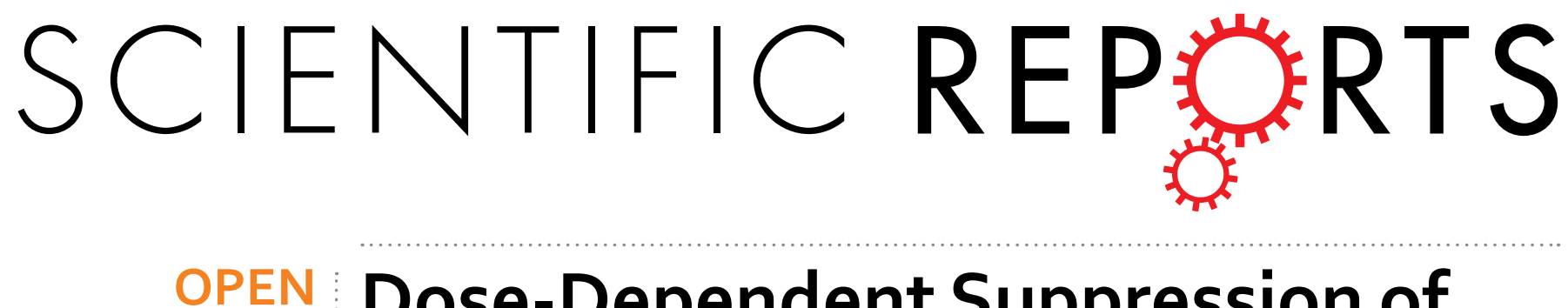

\title{
Dose-Dependent Suppression of Cytokine production from T cells by a Novel Phosphoinositide 3-Kinase Delta Inhibitor
}

Received: 12 April 2016

Accepted: 04 July 2016

Published: 27 July 2016

\author{
Emily E. Way ${ }^{1}$, Giraldina Trevejo-Nunez ${ }^{1}$, Lawrence P. Kane ${ }^{2}$, Bart H. Steiner ${ }^{3}$, Kamal D. Puri ${ }^{3}$, \\ Jay K. Kolls ${ }^{1}$ \& Kong Chen ${ }^{1}$
}

There remains a significant need for development of effective small molecules that can inhibit cytokinemediated inflammation. Phosphoinositide 3 kinase (PI3K) is a direct upstream activator of AKT, and plays a critical role in multiple cell signaling pathways, cell cycle progression, and cell growth, and PI3K inhibitors have been approved or are in clinical development. We examined novel PI3Kdelta inhibitors, which are highly selective for the p110delta is oform of in CD3/CD28 stimulated T-cell cytokine production. In vitro generated CD4+ T effector cells stimulated in the presence of a PI3Kdelta inhibitor demonstrated a dose-dependent suppression of cytokines produced by Th1, Th2, and Th17 cells. This effect was T-cell intrinsic, and we observed similar effects on human PBMCs. Th17 cells expressing a constitutively activated form of AKT were resistant to PI3Kdelta inhibition, suggesting that the inhibitor is acting through AKT signaling pathways. Additionally, PI3Kdelta inhibition decreased IL-17 production in vivo and decreased neutrophil recruitment to the lung in a murine model of acute pulmonary inflammation. These experiments show that targeting PI3Kdelta activity can modulate T-cell cytokine production and reduce inflammation in vivo, suggesting that PI3Kdelta inhibition could have therapeutic potential in treating inflammatory diseases.

The immunologic pathways that lead to inflammation are extremely complex and are, in many cases, incompletely understood. Nevertheless, the components of these pathways are often therapeutic targets for drug development in the treatment of inflammatory conditions. CD4 ${ }^{+} \mathrm{T}$ cells play a central role in many diseases through lineage specific effector cytokine production. To date, three major effector T helper (Th) cell lineages (Th1, Th2 and Th17) have been identified according to their signature cytokines as well as lineage specific transcription factors. Inflammatory pathways involving T helper 17 (Th17) cells have become a particular therapeutic target of interest since their discovery, as these cells have been found to play a vital role in autoimmune and inflammatory conditions, as well as infection ${ }^{1,2}$. Interleukin-17 (IL-17) is a pro-inflammatory cytokine produced by several cell types, including gamma delta $(\gamma \delta)$ T cells and the most recognized and significant of which is the $\mathrm{CD} 4^{+}$effector Th17 cells ${ }^{3,4}$.

Th17 cells differentiate from naïve $\mathrm{CD}^{+} \mathrm{T}$ cells upon stimulation of their T cell receptor (TCR) by antigen and exposure to TGF $\beta$ and IL- 6 in mice, or to IL- $1 \beta$ and IL-23 in humans ${ }^{5}$. The TCR of Th17 cells is composed of an $\alpha$ and $\beta$ chain dimer. During differentiation, the transcription factor Signal Transducers and Activators of Transcription (STAT)-3 stimulates activation of the critical Th17 transcription factor retinoid-related orphan receptor $\gamma \mathrm{t}(\mathrm{ROR} \gamma \mathrm{t})$. ROR $\gamma \mathrm{t}$ is known to promote expression of hallmark Th17 cytokines IL-17A, IL-17F, IL-21, and IL-225. These Th17 cytokines are responsible for the recruitment of several granulopoietic factors including G-CSF, GM-CSF, as well as numerous chemokines and mucins in the process of mediating the immune response to extracellular bacteria and fungi ${ }^{6}$. Th 17 cells play an important role in host defense against infection, but can sometimes be pathogenic when there is dysregulation in the Th17 response and its downstream effector molecules $^{7-9} \cdot \gamma \delta$ T cells, by contrast, express a TCR composed of a $\gamma$ and $\delta$ chain dimer and are typically thought to be involved in stimulation of the adaptive immune response by the innate immune system via driving dendritic

${ }^{1}$ Richard King Mellon Foundation Institute for Pediatric Research, Children's Hospital of Pittsburgh, Pittsburgh, PA, USA. ${ }^{2}$ Department of Immunology, University of Pittsburgh, Pittsburgh, PA, USA. ${ }^{3}$ Gilead Sciences, Inc. Seattle, WA, USA. Correspondence and requests for materials should be addressed to J.K.K. (email: Jay.Kolls@chp.edu) 
cell maturation ${ }^{10} . \gamma \delta \mathrm{T}$ cells are localized to mucosal sites and their activation and production of IL-17 has been shown to be independent of IL-6, but IL-23 and IL-1 $\beta$ dependent ${ }^{11,12}$.

While numerous signaling pathways are involved in T cell differentiation, there has recently been focus on the role of the Phosphatidylinositol 3-Kinase (PI3K) in the differentiation of CD4 ${ }^{+} \mathrm{T}$ cell subsets. PI3K is an enzyme which uses cell membrane lipids to produce second messengers that are involved in numerous cellular functions including growth, survival, protein synthesis, and metabolism ${ }^{13,14}$. PI3Ks are grouped into class I, class II, and class III. Certain class IA PI3K isoforms (PI3K $\alpha$ and $\beta$ ) are expressed ubiquitously in mammalian cells, and are composed of a $110 \mathrm{kDa}$ catalytic subunit and an $85 \mathrm{kDa}$ regulatory subunit ${ }^{13}$. The $\mathrm{p} 110 \delta$ class IA PI $3 \mathrm{~K}$ is preferentially expressed in leukocytes and is crucial for $\mathrm{CD} 44^{+} \mathrm{T}$ cell growth and differentiation ${ }^{14-19}$.

PI3K $\delta$ is activated by receptor tyrosine kinases including the TCR. When the TCR is stimulated by antigen presentation, PI3K $\delta$ is activated through mechanisms which remain unclear but appear to involve TCR costimulatory and adaptor proteins ${ }^{14,20}$. Once activated, PI3K $\delta$ generates phosphatidylinositol-3,4,5-trisphosphate (PIP3) by phosphorylating phosphatidylinositol-4,5-bisphosphate at the plasma membrane. PIP3 then recruits AKT, a serine/threonine kinase also known as Protein Kinase B, to the plasma membrane where it is phosphorylated. $\mathrm{AKT}$, as a major PI3K target, plays a key role in multiple cellular processes such as glucose metabolism, apoptosis, and proliferation. PI3K $\delta$, in particular, plays a critical role in the differentiation process of naïve T cells into effector T helper subsets ${ }^{14,15,20}$, which makes it a strong candidate as a therapeutic target for drug development. There has been marked growth in the development of PI3K inhibitors, though most of these compounds have been aimed at cancer treatment due to the high frequency of dysregulation with this pathway in tumorigenesis ${ }^{21-23}$.

While there is substantial evidence demonstrating that inhibiting PI3K $\delta$ hinders Th 17 differentiation, the more clinically relevant scenario is to inhibit cytokine production from committed Th17 cells after differentiation when they have already become pathogenic. In this study, we examined an isoform-specific inhibitor of PI3K $\delta$ in vitro, with specific focus on the ability of this compound to inhibit production of Th17 cytokines from effector Th17 cells. We demonstrate that the inhibitors, referred to throughout this paper as Compound A for in vitro experiments and Compound B for in vivo experiments, effectively inhibits IL-17 production from Th17 cells but also inhibits cytokine production from other Th lineages. We show that the inhibitor affects $\mathrm{T}$ cells directly and does not require presence of antigen presenting cells in culture. Additionally, we were able to determine that the mechanism through which it affects cytokine production is through inhibition of the AKT signaling pathway.

\section{Results}

Inhibition of PI3K $\delta$ with Compound A results in potent, dose-dependent inhibition of IL-17 production from murine Th17 cells. It has been established by others that inhibition of PI3K $\delta$ results in suppression of Th17 cell differentiation and function and can alleviate inflammation in human and animal models ${ }^{24-27}$. We analyzed the ability of a selective PI3K $\delta$ inhibitor, Compound A, to inhibit IL-17 production from Th17 cells in vitro. Biochemical potency of Compound A (referred as Compound 18 by Patel et al.) is $0.4 \mathrm{nM}$, and selectivity is $200 \mathrm{x}$ or better against the other 3 isoforms ${ }^{28}$. When Th17 cells were differentiated from total OT-II splenocytes and treated with this compound on day three of differentiation, the compound resulted in a potent, dose-dependent inhibition of IL-17 production upon CD3/CD28 stimulation. There was statistically significant IL-17 inhibition even at low concentrations of the PI3K $\delta$ inhibitor (Fig. 1a).

Since the p110 delta subunit of PI3K is most highly expressed in leukocytes ${ }^{17}$, and total splenocytes used in Fig. 1a contain bulk splenocytes, we also verified efficacy of Compound A on isolated T cells. When naïve CD4+/ CD62L + T cells were isolated and then differentiated into Th17 cells over three days, the addition of Compound A demonstrated potent and dose-dependent inhibition of IL-17 production compared to DMSO vehicle control (Fig. 1b). To determine if Compound A affects naïve T cell differentiation into Th1 and/or Th17 lineage in vitro, we added the inhibitor at various concentrations from Day 0 of $\mathrm{T}$ cell polarization. However, the inhibitor seems to have minimal impacts on T cell differentiation (Fig. 1c).

PI3K $\delta$ inhibitor Compound A inhibits cytokine production from multiple Thelper cell types. To determine whether this PI3K $\delta$ inhibitor was specific to TCR activation in Th17 cells, we differentiated Th1 (IL-2, IL-12, and anti-IL4), Th2 (IL-2, IL-4, and anti-IFN $\gamma$ ), and Th17 (IL-6, TGF $\beta$, IL-23, anti-IL4 and anti-IFN $\gamma$ ) cells in vitro for 3 days from naïve CD4 T cells isolated from total splenocytes and treated the cells with the inhibitors upon CD3/CD28 re-stimulation. Luminex on the supernatants of these cells showed significant inhibition of interferon- $\gamma($ IFN $\gamma)$ from Th1 cells, IL- 5 from Th2 cells, and continued to show inhibition of IL-17 production from Th17 cells (Fig. 2). Of note, 7-AAD staining was performed on these cells after treatment with Compound A as well as DMSO, and there was no cell toxicity at these concentrations to explain the level of cytokine inhibition (data not shown).

Compound A directly inhibits AKT function. Because T cell development and differentiation depends on the canonical pathway for PI3K activation in which PIP3 recruits AKT to the plasma membrane resulting in its phosphorylation and activation ${ }^{15}$, we hypothesized that inhibition of AKT would also result in IL-17 inhibition. Naïve T cells were isolated from mouse splenocytes and treated with Compound A or a commercially available inhibitor of AKT isoforms 1 and 2 on day 3 of differentiation. Cells were then stimulated on day 5 with CD3/ CD28 stimulation. We observed inhibition of IL-17 production with both inhibitors (Fig. 3) consistent with the notion that both $\mathrm{PI} 3 \mathrm{~K} \delta$ and $\mathrm{AKT}$ are required for CD3/CD28 stimulated IL-17 production.

To confirm that this PI3K $\delta$ inhibitor is indeed acting through the AKT pathway, we transduced murine Th17 cells with a retrovirus expressing myristoylated $\mathrm{AKT}^{29}$, which is a constitutively active form of AKT (Fig. 4a). Once these Th17 cells were transduced, they were sorted by flow cytometry into a GFP negative population expressing wild type AKT and a GFP positive population expressing myristoylated AKT (Fig. 4b). When these Th17 cells were treated with AKT inhibitor or Compound A prior to CD3/CD28 stimulation, the GFP positive 
a)

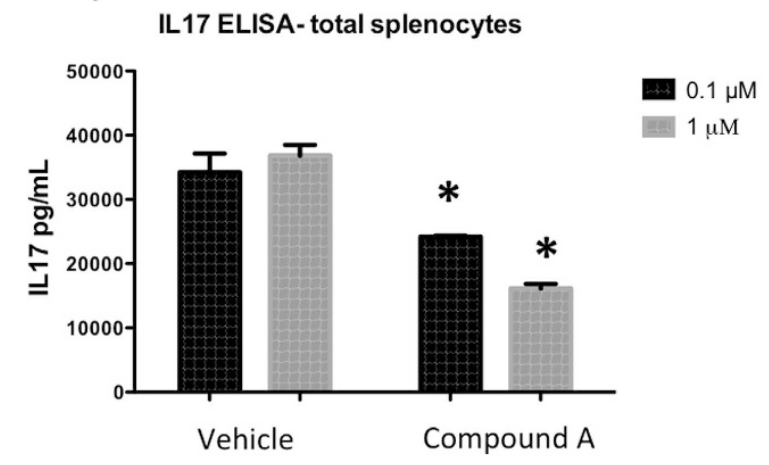

b)

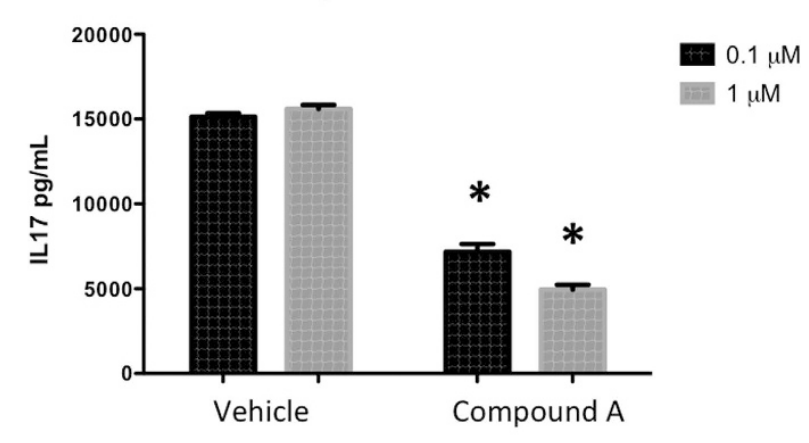

c)
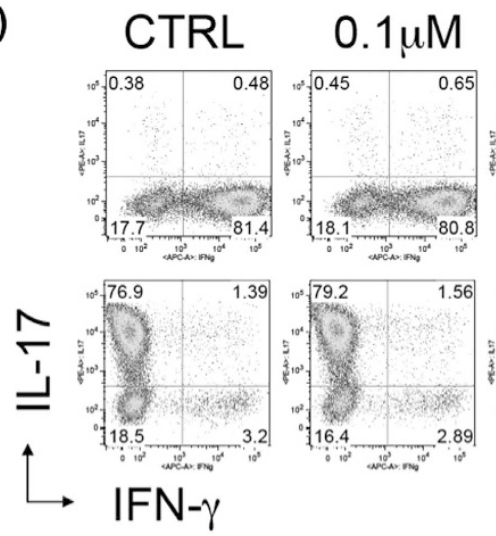
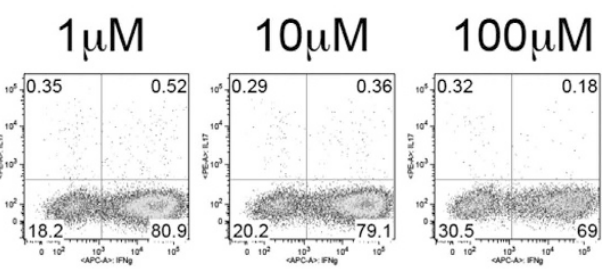

Th1
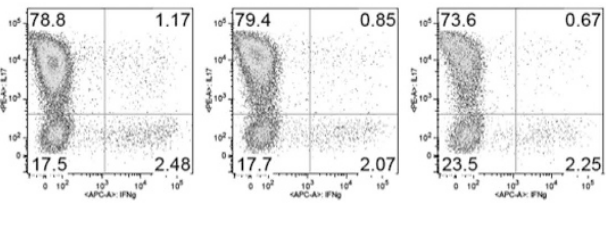

Th17

Figure 1. Inhibiting PI3K $\delta$ results in potent, dose-dependent suppression of IL-17 production from total splenocytes and isolated Th17 cells. (a) Transgenic OT II mouse total splenocytes, which contain T cells that are specific to ovalbumin peptide, were differentiated into Th17 cells and treated with inhibitor compounds at two different concentrations on day 3 of differentiation. Compared to vehicle control, the PI3K $\delta$ inhibitor results in significant inhibition of IL-17 production in a dose-dependent manner as measured by ELISA (b) CD4+/ $\mathrm{CD} 62 \mathrm{~L}+$ naïve $\mathrm{T}$ helper cells were isolated from an IL-17F reporter mouse which uses Thy1.1 expression as a marker for IL-17F production, and then differentiated into Th17 cells. Th17 cells were then further purified by sorting thy $1.1+$ cells on flow cytometer and then treated with inhibitor compounds on day 3 of differentiation. Compared to vehicle control, the PI3K $\delta$ inhibitor results in significant inhibition of IL-17 production in a dose-dependent manner as measured by ELISA. * $\mathrm{p}<0.05$, compared to DMSO (c) Transgenic OT II mouse total splenocytes, which contain T cells that are specific to ovalbumin peptide, were differentiated into Th1 and Th17 cells and treated with inhibitor compounds at two concentrations on day 1 of differentiation. Effects of the PI3K $\delta$ inhibitor on $\mathrm{T}$ cell polarization were analyzed by intracellular staining by FACS.

cells that contain constitutively active AKT were resistant to the effects of Compound A, while the GFP negative cells that contain wild type AKT continue to demonstrate potent, dose-dependent inhibition of IL-17 production (Fig. 4c). Taken together, these data demonstrate that the mechanism through which CD3/CD28 stimulated IL-17 production is inhibited in Th17 cells by Compound A is through suppressing AKT activation.

Compound B is a Potent and Selective Inhibitor of PI3K $\delta$. A novel selective PI3K $\delta$ inhibitor, Compound B, that had potency, demonstrable oral bioavailability and adequate plasma half-life to evaluate its activity in vivo in mice, was utilized. Compound B (GS-649443) is structurally related to Compound A and share the same core (manuscript in preparation). However, Compound B offers superior mouse pharmacokinetics over Compound $\mathrm{A}$, and was therefore preferred for the in vivo efficacy studies. In a FRET-based in vitro kinase assay, the Compound B inhibited PI3K $\delta$ with an $\mathrm{IC}_{50}$ of $0.28 \mathrm{nM}$ and it was 87 - to 4150 -fold more selective over the other PI3K class I enzymes (manuscript in preparation). In addition, Compound B was also $>2500$-fold more selective for PI3K $\delta$ over other related kinases, such as $\mathrm{CII} \alpha, \mathrm{CII} \beta, \mathrm{hVPS} 34, \mathrm{PI} 4 \mathrm{~K} \alpha, \mathrm{mTOR}, \mathrm{PIP} 5 \mathrm{~K} 1 \alpha$, and PIP5K2 $\beta$ (manuscript in preparation).

Although Compound B selectivity for the PI3K $\delta$ Class I PI3K isoform was shown by $\mathrm{IC}_{50}$ determinations, we tested Compound B binding to kinases outside the PI3K family using a panel of 456 kinases (KinomeScan; DiscoveRx $)^{30}$. A high Compound B concentration $(10 \mu \mathrm{M})$ in a KinomeScan assay was used. Compound B only 
a)

IFN $\gamma @ 1 \mu M$ Inhibitor

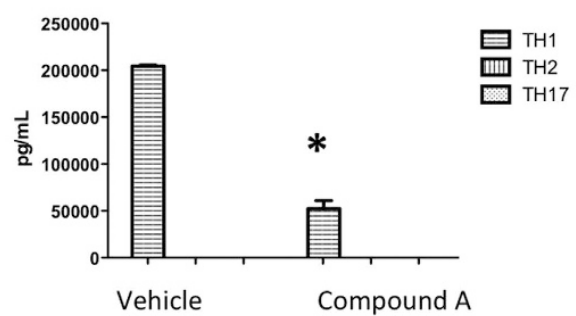

c) b)

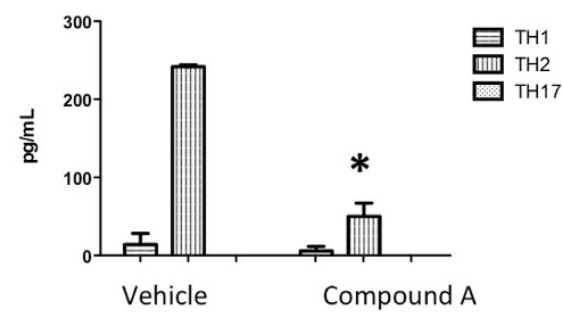

IL17 @1 $\mu$ M Inhibitor

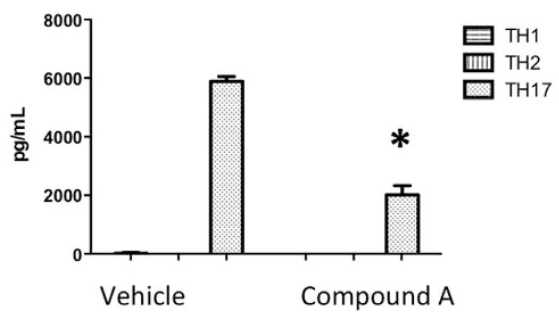

Figure 2. Inhibiting PI $3 K \delta$ results in potent, dose-dependent suppression of cytokine production from all three major T helper cell lineages. Transgenic DO.11.10 mouse splenocytes, which contain T cells that are specific to ovalbumin peptide, were differentiated into (a) Th1, (b) Th2, and (c) Th17 cells and treated with inhibitor compounds at $1 \mu \mathrm{M}$ concentration on day 3 of differentiation. Compared to vehicle control, inhibiting PI3K $\delta$ results in suppression of hallmark cytokine production from all three cell types, indicating that this compound is not specific to Th17 cells. *p $<0.05$, compared to DMSO.

\section{IL17 ELISA- purified Th17 cells}

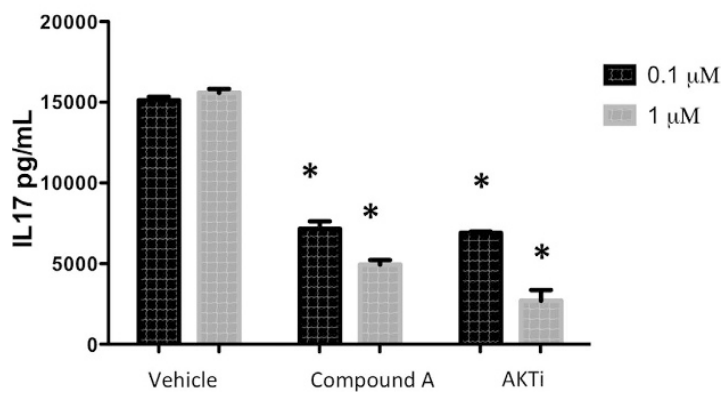

Figure 3. Inhibiting PI $3 K \delta$ results in similar suppression of IL-17 production as AKT inhibition from isolated Th17 cells. CD4+/CD62L + naïve T helper cells were isolated from an IL-17F reporter mouse which uses Thyl.1 expression as a marker for IL-17F production and then differentiated into Th17 cells. Th17 cells were then further isolated by sorting thyl.1+ cells on flow cytometer and then treated with inhibitor compounds on day 3 of differentiation. Compared to vehicle control, the PI $3 \mathrm{~K} \delta$ inhibitor results in strikingly similar inhibition of IL-17 production as an AKT inhibitor. *p $<0.05$, compared to DMSO.

binds class I PI3K (Fig. 5, Supplemental Table 1) and inhibits PI $3 \mathrm{~K} \delta$ at an $\mathrm{IC}_{50}$ of $\sim 0.28 \mathrm{nM}$, further demonstrating a high degree of selectivity for PI3K $\delta$.

Compound B inhibits human T-cell cytokine production. The ability of Compound B to inhibit TCR-stimulated cytokine release was evaluated in normal human PBMCs. Isolated PBMCs in serum-free AIM-V media were treated with either vehicle or Compound B followed by stimulation by CytoStim reagent. The production of cytokines was evaluated by Meso Scale Discovery assays after 48 hours of stimulation. Compound B inhibited TNF $\alpha$, IFN $\gamma$, IL-4, IL-5, IL-10, IL-12 p70, IL-13, IL-17, and IL-1 $\beta$ secretion in human PBMCs with geometric mean EC50 values of 0.29 to $1.0 \mathrm{nM}$, demonstrating that Compound B inhibits TCR-triggered signaling that leads to secretion of these inflammatory cytokines (Fig. 6a). Production of IL-2 was only modestly inhibited and IL-8 production was not inhibited. The individual $\mathrm{EC}_{50}$ values are listed (Fig. $6 \mathrm{~b}$ ) and these values are much lower that the EC50 values (Table 1) of Idelalisib (GS-1101, CAL-101), a potent and selective small molecule inhibitor of the delta isoform of the p110 subunit PI3K (PI3K p1108) recently approved for the treatment of chronic lymphocytic leukemia and non-Hodgkin lymphoma ${ }^{31-33}$. Cell viability from 4 donors was not significant altered by increasing 
a)

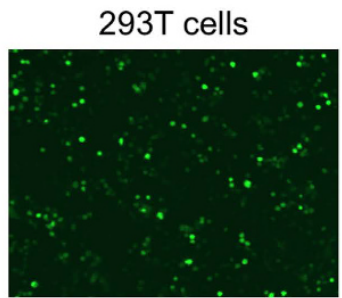

Th17 cells

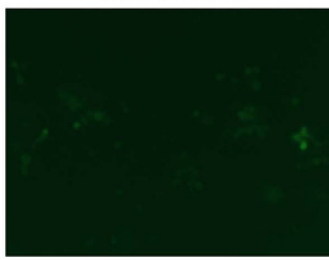

c)

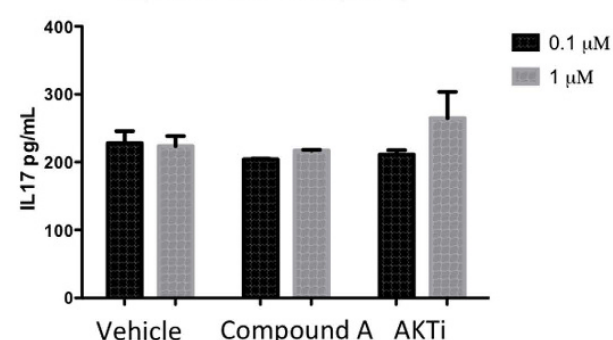

b)
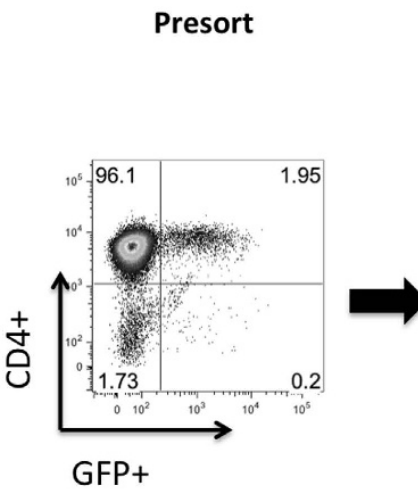

Postsort

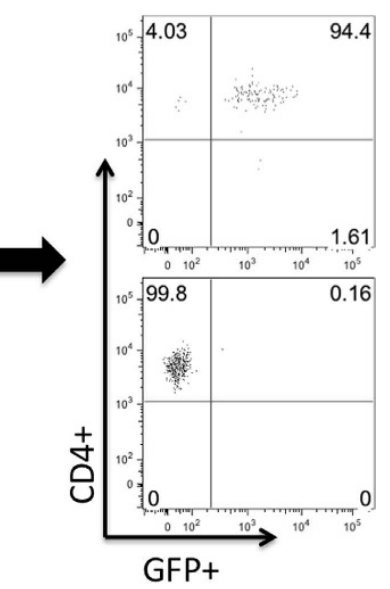

WT-AKT Th17 cells (GFP-)

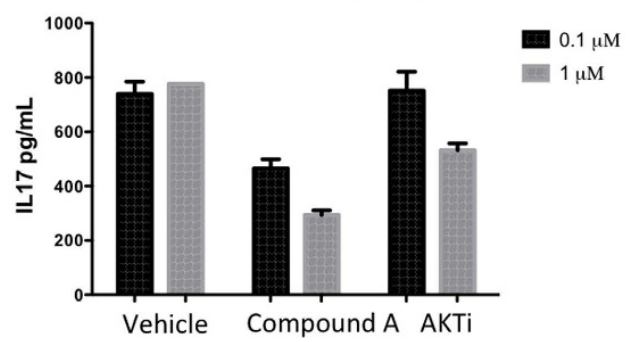

Figure 4. Th17 cells transfected with constitutively active AKT are resistant to effects of PI3K $\delta$ inhibition compared to Th17 cells containing wild type AKT (a) 293T cells were transduced with a plasmid containing GFP and myristoylated AKT as well as retroviral packaging plasmid (left). Mouse Th17 cells, derived from OT II splenocytes, were then transfected with retrovirus (right). (b) After retroviral transduction, splenocytes were sorted into GFP + and GFP- populations. (c) OT II mouse splenocytes were treated with PI3K $\delta$ inhibitor and AKT inhibitor. GFP+ Th17 cells, which contain constitutively active AKT, do not demonstrate IL-17 inhibition when treated with PI3K $\delta$ inhibitor while GFP- Th17 cells, which contain wild type AKT, continue to demonstrate potent inhibition of IL-17 production. ${ }^{*} \mathrm{p}<0.05$.

the concentration of compound B (Fig. 6c). These data suggest that Compound B is more potent than Idelalisib. This is of clinical significance as increasing the potency could reduce the dose level and may limit dose-associated toxicities.

Inhibition of PI3K $\delta$ results in reduced IL-17 mediated inflammation. While PI3K $\delta$ is known to play an important role in IL-17 production by CD $4+\mathrm{T}$ cells, its role in IL-17 production by $\gamma \delta \mathrm{T}$ cells is less clear. However, $\gamma \delta \mathrm{T}$ cells have been shown to play a significant role in the pathogenesis of autoimmune diseases ${ }^{34}$, and thus inhibiting PI3K $\delta$ could be therapeutically beneficial if it does result in inhibition of $\gamma \delta \mathrm{T}$ cell function. We sought to determine whether inhibiting PI $3 \mathrm{~K} \delta$ in pulmonary $\gamma \delta \mathrm{T}$ cells could also result in decreased IL-17 production. To examine this we administered Compound B by oral gavage to mice that were treated with intranasal IL-1 $\beta$ and IL-23 which activates pulmonary $\gamma \delta$ T cells to express IL-17 (Fig. 7a) ${ }^{35,36}$. The lungs of mice that were treated with the experimental compound demonstrated $50 \%$ reduced expression of IL-17A by real time PCR compared with mice that received vehicle control (Fig. 7b). Additionally, there was also $50 \%$ reduction of pulmonary IL-17 at the protein level in broncheoalveolar lavage (BAL) fluid of mice treated with the PI3K $\delta$ inhibitor (Fig. 7c). To further evaluate the effect of the experimental compound on the inflammatory cell infiltrate in the lung alveolar space, we performed FACS analysis on the BAL cells. Consistent with suppression of IL-17, neutrophils were selectively decreased as we expected in the BAL fluid of mice treated with Compound B while percentages of macrophages and total cells in the BAL fluid were not statistically different between groups (Fig. 7d). To determined the cellular sources of IL-17, we performed intracellular cytokine staining on the mononuclear cells isolated from the lungs of mice received the treatment of Compound B. Both $\gamma \delta-\mathrm{T}$ and CD $4^{+} \mathrm{T}$ cells appeared to be IL-17 producers in this model and the IL-17 production was suppressed by Compound B in both cell populations (Fig. 7e).

\section{Discussion}

In this study, we have shown that Compound A, a novel isoform-specific inhibitor of PI3K $\delta$, has a potent and dose-dependent inhibitory effect on cytokine production from Thelper cells when they are stimulated through TCR and CD28 activation. It inhibited IL-17 production from Th17 cells, and also inhibited production of key cytokines by murine and human Th1 and Th2 cells after stimulation. This non-specific effect on all three major 


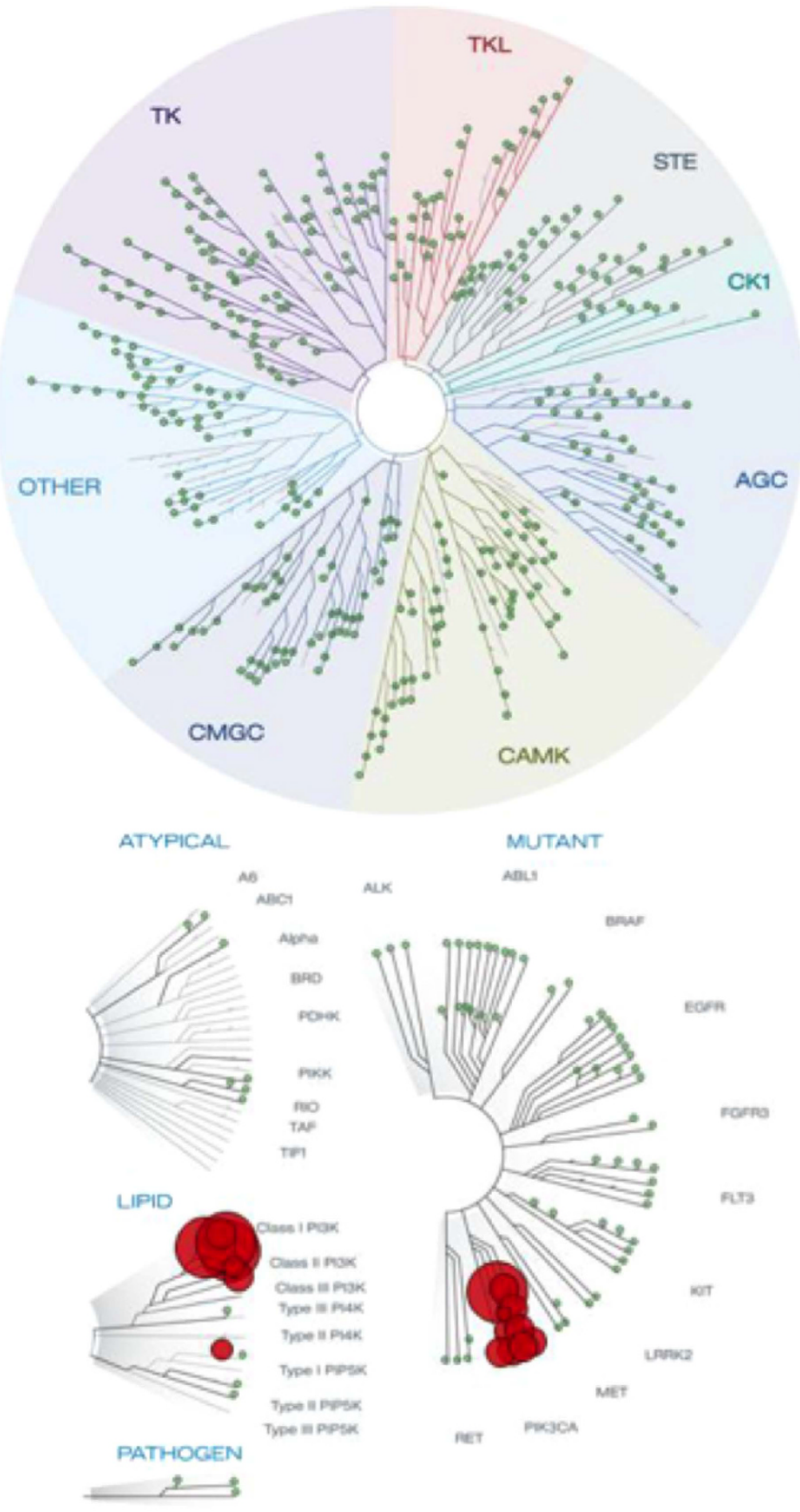

Figure 5. Compound B binds selectively to class I PI3K. Compound B specificity was tested in a kinaseselectivity profiling assay on a panel of 456 kinases. Compound B $(10 \mu \mathrm{M})$ was tested in an ATP site competition assay. Compound $\mathrm{B}$ was considered active for a kinase when the kinase fraction that remained bound to ATP was less than $30 \%$. Red circles indicate the kinases for which Compound B shows binding activity. Compound B showed no significant binding activity outside the PI3K family.

T helper cell subsets is not surprising, as there is no evidence that the delta isoform of PI3K is differentially expressed among various $\mathrm{T}$ helper cell lineages. In fact, the broad-spectrum suppression of various Th effectors may be beneficial in clinical setting since many inflammatory diseases such as severe asthma and rheumatoid arthritis are characterized by combinations of Th1, Th2 and/or Th17 driven immune responses ${ }^{37,38}$.

The mechanism by which Compound A inhibited cytokine production was through attenuating the AKT signaling pathway. This pathway is known to regulate differentiation of Th17 cells by AKT activation of the 
a)
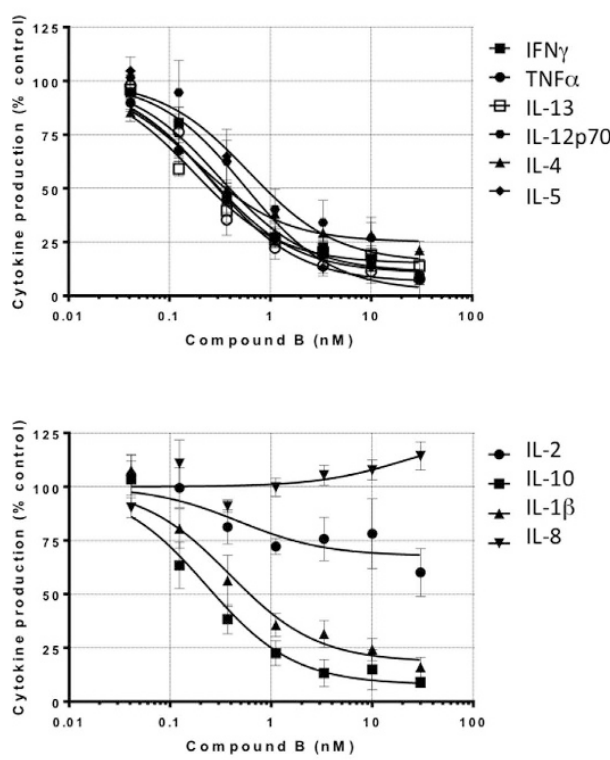

b)

\begin{tabular}{|l|l|}
\hline Cytokine & $\begin{array}{l}\text { Compound } \\
\text { B } \\
\mathrm{EC}_{50} \text { (nM), } \\
\text { n=4 }\end{array}$ \\
\hline TNF $\alpha$ & 0.35 \\
\hline IFNY & 0.43 \\
\hline IL-4 & 0.52 \\
\hline IL-5 & 0.58 \\
\hline IL-10 & 0.29 \\
\hline IL-12 p70 & 1.0 \\
\hline IL-13 & 0.29 \\
\hline IL-17 & 0.32 \\
\hline IL-1 $\beta$ & 0.74 \\
\hline IL-2 & $>30$ \\
\hline IL-8 & $>30$ \\
\hline
\end{tabular}

c)

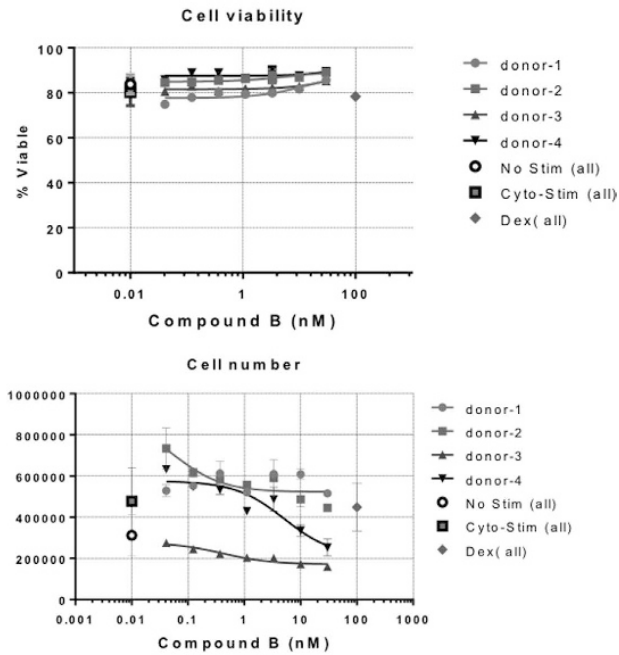

Figure 6. Compound B inhibits Th1 and Th2 cytokine secretion by activated T cells. Cytokines produced by activated $\mathrm{T}$ cells were analyzed using human PBMC preincubated with serial dilutions of Compound $\mathrm{B}(48 \mathrm{~h})$ and activated via anti-CD3/MHC-II (see Methods). Cytokine levels in culture supernatants were assessed using MSD assay plate (Meso Scale Discovery, Gaithersburg, MD). Graphs show percent inhibition of cytokine production at different Compound B concentrations compared to maximum cytokine levels (without Compound $\mathrm{B} ; 100 \%)$. The table shows the apparent $\mathrm{IC}_{50}$ for the inhibitor.

\begin{tabular}{|c|c|c|c|c|}
\hline Cell Type & Stimuli (Receptor) & Assay & Readout & $\mathrm{EC}_{50}(\mathrm{nM})$ \\
\hline \multirow{9}{*}{ T cells } & \multirow{9}{*}{ Anti-CD3/Anti-MHC II (TCR/MHC II) } & \multirow{9}{*}{ Cytokines release } & IL-4 & 2.4 \\
\hline & & & IL-5 & 2.7 \\
\hline & & & IL-13 & 3.1 \\
\hline & & & IL-17 & 3.5 \\
\hline & & & TNF $\alpha$ & 5.2 \\
\hline & & & IFN $\gamma$ & 4.8 \\
\hline & & & IL-1 $\beta$ & 6.1 \\
\hline & & & IL-12p70 & 2.3 \\
\hline & & & IL-10 & 2.6 \\
\hline
\end{tabular}

Table 1. In Vitro Potency of Idelalisib on Human T-Cell Cytokine Production.

Mechanistic Target of Rapamycin Complex 1 (mTORC1), thereby activating HIF-1 $\alpha$ and S6K2, as well as inhibiting Gfi $1^{16,18,20}$. There is some debate over whether inhibition of PI3K $\delta$ affects Th 17 differentiation by inducing FoxP3 expression, but the evidence supporting this mechanism is mainly found in experiments where PI3K $\delta$ expression is modified prior to T helper cell differentiation ${ }^{18,19,39,40}$. For the purposes of this paper, we focused on inhibiting PI $3 \mathrm{~K} \delta$ after differentiation of these effector T helper cells, as this is more analogous to true disease states in which effector $\mathrm{T}$ cells have already formed.

Since $\gamma \delta$ T cells have been implicated in the pathogenesis of autoimmune diseases ${ }^{34}$, evaluation as to whether PI3K $\delta$ inhibition could inhibit IL-17 production from $\gamma \delta \mathrm{T}$ cells would be impactful. We used experimental Compound $\mathrm{B}$, which is a $\mathrm{PI} 3 \mathrm{~K} \delta$ inhibitor structurally-related to Compound $\mathrm{A}$, with more suitable pharmacokinetic properties for an in vivo mouse model of pulmonary inflammation. This study demonstrates that pulmonary $\gamma \delta \mathrm{T}$ cell function is affected by PI3K $\delta$ inhibition in that IL-17 was decreased following stimulation with IL-1 $\beta$ and IL-23 and this results in reduced neutrophil infiltrates in BAL fluid. This is likely a reflection of decreased neutrophil recruitment to the lung in these mice treated with experimental compound, as inhibition of IL-17 may result in attenuated activation of its downstream chemokines, which are responsible for neutrophil recruitment ${ }^{41}$. There was a trend toward decreased expression of Cxcll and Csf3 mRNA in the mice treated with experimental compound, but this data did not reach statistical significance (data not shown). Since CXCL1 acts as a neutrophil chemoattractant while G-CSF plays more of a role in neutrophil mobilization, the ultimate effect of decreased neutrophil recruitment in this experiment may have been additive or synergistic from the combination of the effects of decreased Cxcl1 and Csf3 ${ }^{42-44}$. 
a) Timeline of in vivo experiments

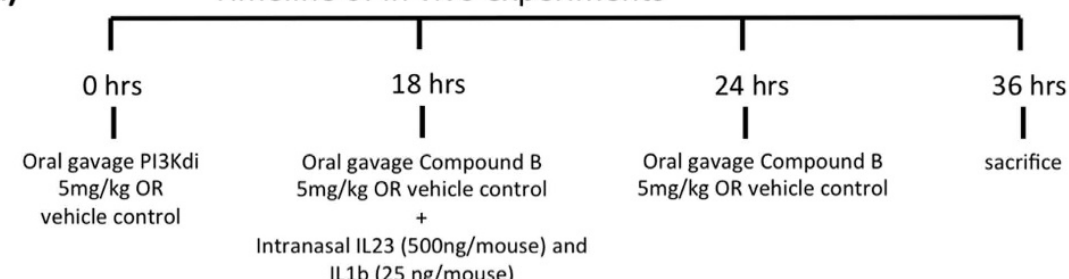

b)

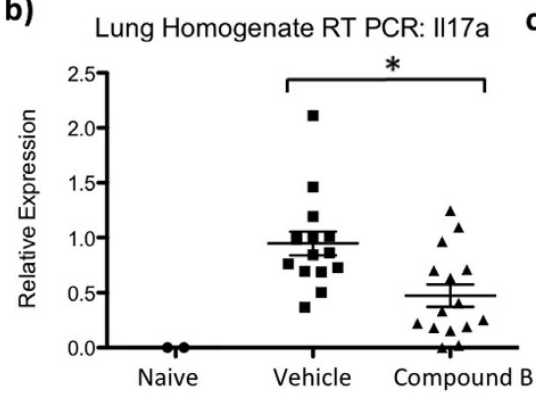

e)

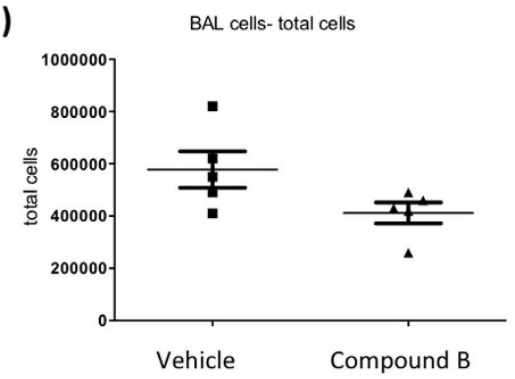

c)

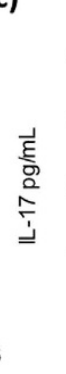

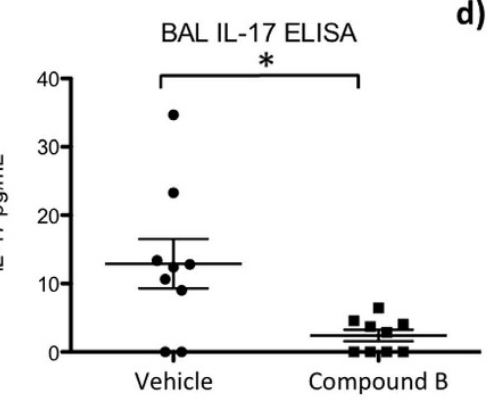

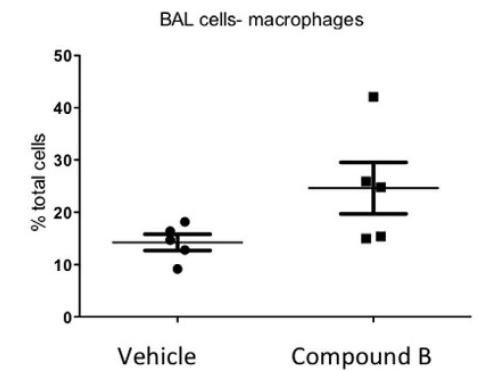

d)
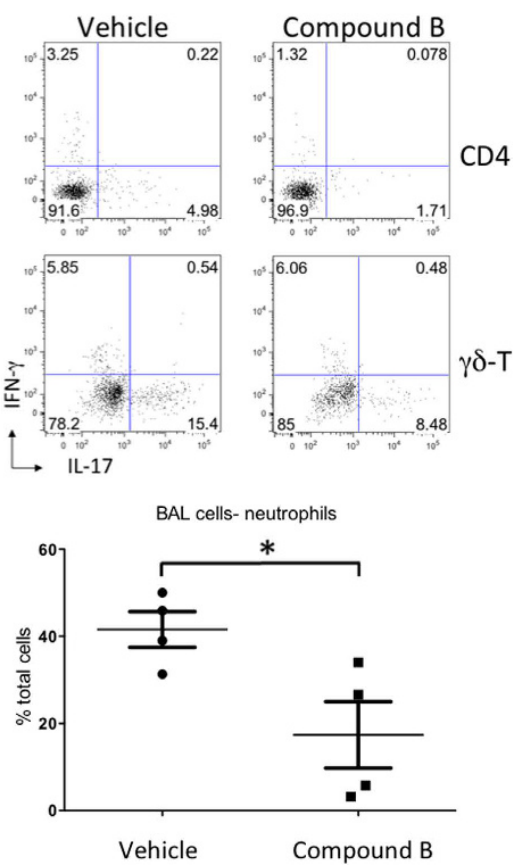

Figure 7. In vivo Inhibition of PI3K $\delta$ in mice results in decreased IL-17 production by Gamma Delta T cells. (a) B6 mice were treated with Compound B or vehicle control by oral gavage. All mice received intranasal IL-1b and IL-23 to activate $\gamma \delta$ T cells at indicated time points. (b) Real time PCR demonstrates a significant reduction in IL-17a expression in the lung homogenate of mice treated with Compound B. Naïve mice that did not receive intranasal IL-1b or IL-23 did not express any IL-17a. This is pooled data from 3 independent experiments. (c) IL-17 ELISA shows a significantly decreased IL-17 levels in the BAL fluid in mice treated with Compound B. This is pooled data from 2 independent experiments. (d) Representative intracellular cytokine staining FACS plots for IL-17 and IFN- $\gamma$ gated on both $\gamma \delta$-T cells and CD $4^{+} \mathrm{T}$ cells from the lung mononuclear cells were shown $(\mathrm{N}=4-5)$. (e) Flow cytometry showed a significantly lower percentage of neutrophils in the BAL fluid of mice treated with experimental compound while there were no significant differences in total cells or percentage of macrophages between groups. This data is representative of 3 independent experiments.

Mutations and dysregulation in the PI3K/AKT pathways are heavily involved in cancer pathogenesis, and thus this pathway is highly targeted in cancer research. The PI $3 \mathrm{~K} \delta$ isoform specifically has been shown to be abnormally expressed or abnormally signal in several malignancies and small molecule inhibitors of this isoform are in clinical trials for cancer treatment and one is FDA approved for the treatment of CLL ${ }^{23,45}$. However, given the preferential expression of this isoform in leukocytes, it is more recently being investigated as a therapeutic target in nonmalignant inflammatory conditions as well. Inhibition of PI3K $\delta$ is known to suppress Th2 inflammatory responses from PBMCs taken from human patients with environmental allergies and suppresses IFN $\gamma$, IL-17, and TNF- $\alpha$ production by PBMCs from human patients with rheumatoid arthritis ${ }^{26}$. PI3K $\delta$ kinase-dead knock-in mice demonstrate reduced skin inflammation in psoriasis models, and PBMCs from human psoriasis patients have lower ex vivo stimulated IL-17 production when treated with PI3K $\delta$ inhibitors in cell culture ${ }^{27}$. A gain-of-function mutation in the PI $3 \mathrm{~K} \delta$ isoform has also been discovered which is associated with recurrent respiratory infections and airway damage, highlighting the importance of this molecule in inflammatory diseases ${ }^{46}$.

There are currently a myriad of treatment options available for patients with autoimmune inflammatory conditions including antimetabolites, calcineurin inhibitors, monoclonal antibodies to specific cytokines and their receptors, B cell inhibitors, and even alkylating agents. However, for all of the successes that each therapy has experienced, there are always patients who are refractory and require change to or addition of other agents. Therefore, there is a need for new classes of medications for the treatment of autoimmune inflammatory conditions; PI3K $\delta$ inhibitors could have an important role in the treatment of these conditions and these molecules are currently being evaluated for treatment of several inflammatory disorders. 


\section{Materials and Methods}

Animals. 4-8 week old female OT-II and DO11.10 mice were obtained from Jackson Lab (Bar Harbor, ME). Thy1.1 IL-17F reporter mice, originally developed in the lab of Casey Weaver at University of Alabama Birmingham ${ }^{47}$, were bred in-house at The University of Pittsburgh. All animal studies were approved by the Institutional Animal Care and Use Committee (IACUC) of the University of Pittsburgh. All experiments were performed in accordance with IACUC guidelines and regulations. 8-10 week old female C57BL/6 mice from Jackson lab were used for in vivo studies. On day 0 they were given $5 \mathrm{mg} / \mathrm{kg}$ Compound B) by oral gavage or same volume of vehicle control. Compound B or vehicle control was dosed every 12-18 hours for 3 doses on day 0 and day 1 . On day 1, 24 hours before sacrificing, every mouse received Intranasal IL-23 (500 ng/mouse) and IL-1 $\beta$ ( $25 \mathrm{ng} /$ mouse) to induce gamma-delta T cell activation ${ }^{35,36}$. On Day 2 the mice were sacrificed, BAL was performed, and lungs were harvested. All animal studies were approved by the Institutional Animal Care and Use Committee of the University of Pittsburgh.

T cell differentiation. Total splenocytes or naive CD4+/CD62L + cells were incubated for 3 days in the presence of IL-2 (50 units $/ \mathrm{mL})$, IL-12 $(10 \mathrm{ng} / \mathrm{mL})$, and anti-IL4 $(5 \mu \mathrm{g} / \mathrm{mL})$ for Th1 cell differentiation; IL-2 (50 units $/ \mathrm{mL})$, IL-4 $(10 \mathrm{ng} / \mathrm{mL})$, and anti-IFN $\gamma(5 \mu \mathrm{g} / \mathrm{mL})$ for Th2 cell differentiation; or IL-6 $(40 \mathrm{ng} / \mathrm{mL})$, TGF $\beta(5 \mathrm{ng} / \mathrm{mL})$, IL-23 $(10 \mathrm{ng} / \mathrm{mL})$, anti-IL4 $(5 \mu \mathrm{g} / \mathrm{mL})$ and anti-IFN $\gamma(5 \mu \mathrm{g} / \mathrm{mL})$ for Th17 cell differentiation. All recombinant mouse cytokines were purchased from R\&D Systems, Inc (San Diego, CA) and eBioscience. OT-II and DO11.10 splenocytes were activated by chicken ovalbumin peptide, OVA (323-339), (AnaSpec, Fremont, CA) and naïve CD4+/CD62L + T cells were activated by plate-bound anti-CD3 and anti-CD28 antibodies from eBioscience, Inc. Naïve CD4+/CD62L+ T cells were isolated by MACS beads from Miltenyi Biotec (Auburn, CA).

Cytokine Inhibitor Compounds. Compounds were reconstituted at $10 \mathrm{mM}$ with DMSO and diluted to $1 \mu \mathrm{M}$, and $0.1 \mu \mathrm{M}$ for cell culture. Cells are washed twice prior to addition of inhibitors. 30-60 minutes after addition of inhibitors, T cells were re-stimulated with anti-CD3/anti-CD28 coated beads (Life Technologies, Grand Island, NY). Cells are incubated with inhibitor compounds and beads for 18-24 hours. AKT 1/2 inhibitor (cat\# 612847-09-03) was purchased from EMD Millipore (Billerica, MA).

T cell transduction. Th17 cells were differentiated from OT-II splenocytes while 293T cells were transduced with myrAKT plasmid and pCL-ECO packaging plasmid (Biomol, Hamburg, Germany) using Polyethyleneimine and $\mathrm{NaCl}$. On day 3 of $293 \mathrm{~T}$ cell transduction and Th17 cell differentiation, retroviral supernatant was harvested and Th17 cells were centrifuged in a 24 -well plate with $1 \mathrm{~mL}$ viral supernatant/well and polybrene $10 \mu \mathrm{g} / \mathrm{mL}$ at $300 \mathrm{~g}$ for $1 \mathrm{hr}$. On day 4, retroviral supernatant was harvested again and centrifugation repeated. Th17 cells were restimulated with chicken ovalbumin peptide after each round of transduction, then incubated in IMDM with TGF $\beta$, IL-6, and IL-23 for 5 additional days. Th17 cells were then sorted into CD4+/GFP+ and CD4+/ GFP- populations.

Statistics. All data are presented as the mean \pm SEM. For comparison between two groups, student $t$-test was used. A P-value of less than 0.05 was considered significant.

\section{References}

1. Miossec, P. \& Kolls, J. K. Targeting IL-17 and TH17 cells in chronic inflammation. Nature reviews. Drug discovery 11, 763-776 (2012).

2. Robinson, K. M., Manni, M. L., Biswas, P. S. \& Alcorn, J. F. Clinical Consequences of Targeting IL-17 and T17 in Autoimmune and Allergic Disorders. Current allergy and asthma reports (2013).

3. Harrington, L. E. et al. Interleukin 17-producing CD4+ effector T cells develop via a lineage distinct from the Thelper type 1 and 2 lineages. Nature immunology 6, 1123-1132 (2005).

4. Park, H. et al. A distinct lineage of CD4 T cells regulates tissue inflammation by producing interleukin 17. Nature immunology 6, 1133-1141 (2005).

5. McGeachy, M. J. \& Cua, D. J. Th17 cell differentiation: the long and winding road. Immunity 28, 445-453 (2008).

6. Ouyang, W., Kolls, J. K. \& Zheng, Y. The biological functions of T helper 17 cell effector cytokines in inflammation. Immunity 28, 454-467 (2008).

7. Curtis, M. M. \& Way, S. S. Interleukin-17 in host defence against bacterial, mycobacterial and fungal pathogens. Immunology 126, $177-185$ (2009).

8. Khader, S. A., Gaffen, S. L. \& Kolls, J. K. Th17 cells at the crossroads of innate and adaptive immunity against infectious diseases at the mucosa. Mucosal immunology 2, 403-411 (2009).

9. Way, E. E., Chen, K. \& Kolls, J. K. Dysregulation in lung immunity - The protective and pathologic Th17 response in infection. European journal of immunology (2013).

10. Ismaili, J., Olislagers, V., Poupot, R., Fournie, J. J. \& Goldman, M. Human gamma delta T cells induce dendritic cell maturation. Clinical immunology 103, 296-302 (2002).

11. Sutton, C. E. et al. Interleukin-1 and IL-23 induce innate IL-17 production from gammadelta T cells, amplifying Th17 responses and autoimmunity. Immunity 31, 331-341 (2009).

12. Lochner, M. et al. In vivo equilibrium of proinflammatory IL-17+ and regulatory IL-10+ Foxp3+ RORgamma $\mathrm{t}+\mathrm{T}$ cells. J Exp Med 205, 1381-1393 (2008).

13. Vanhaesebroeck, B. et al. Synthesis and function of 3-phosphorylated inositol lipids. Annual review of biochemistry 70, 535-602 (2001).

14. Okkenhaug, K. \& Vanhaesebroeck, B. PI3K in lymphocyte development, differentiation and activation. Nature reviews. Immunology 3, 317-330 (2003).

15. Han, J. M., Patterson, S. J. \& Levings, M. K. The Role of the PI3K Signaling Pathway in CD4(+) T Cell Differentiation and Function. Frontiers in immunology 3, 245 (2012).

16. Nagai, S., Kurebayashi, Y. \& Koyasu, S. Role of PI3K/Akt and mTOR complexes in Th17 cell differentiation. Annals of the New York Academy of Sciences 1280, 30-34 (2013). 
17. Kok, K., Geering, B. \& Vanhaesebroeck, B. Regulation of phosphoinositide 3-kinase expression in health and disease. Trends in biochemical sciences 34, 115-127 (2009).

18. Kurebayashi, Y. et al. PI3K-Akt-mTORC1-S6K1/2 axis controls Th17 differentiation by regulating Gfil expression and nuclear translocation of RORgamma. Cell reports 1, 360-373 (2012).

19. Kim, J. S. et al. Natural and inducible TH17 cells are regulated differently by Akt and mTOR pathways. Nature immunology 14, 611-618 (2013).

20. Okkenhaug, K. et al. The p110delta isoform of phosphoinositide 3-kinase controls clonal expansion and differentiation of Th cells. J Immunol 177, 5122-5128 (2006).

21. Massacesi, C., di Tomaso, E., Fretault, N. \& Hirawat, S. Challenges in the clinical development of PI3K inhibitors. Annals of the New York Academy of Sciences 1280, 19-23 (2013).

22. Rodon, J., Dienstmann, R., Serra, V. \& Tabernero, J. Development of PI3K inhibitors: lessons learned from early clinical trials. Nature reviews. Clinical oncology 10, 143-153 (2013).

23. Tzenaki, N. \& Papakonstanti, E. A. p110delta PI3 kinase pathway: emerging roles in cancer. Frontiers in oncology 3, 40 (2013).

24. Park, S. J. et al. Phosphoinositide 3-kinase delta inhibitor suppresses interleukin-17 expression in a murine asthma model. The European respiratory journal 36, 1448-1459 (2010).

25. Haylock-Jacobs, S. et al. PI3Kdelta drives the pathogenesis of experimental autoimmune encephalomyelitis by inhibiting effector T cell apoptosis and promoting Th17 differentiation. Journal of autoimmunity 36, 278-287 (2011).

26. Soond, D. R. et al. PI3K p110delta regulates T-cell cytokine production during primary and secondary immune responses in mice and humans. Blood 115, 2203-2213 (2010).

27. Roller, A. et al. Blockade of phosphatidylinositol 3-kinase PI3Kdelta or PI3Kgamma reduces IL-17 and ameliorates imiquimodinduced psoriasis-like dermatitis. J Immunol 189, 4612-4620 (2012).

28. Patel, L. et al. 2,4,6-Triaminopyrimidine as a Novel Hinge Binder in a Series of PI3Kdelta Selective Inhibitors. J Med Chem 59, 3532-3548 (2016).

29. Kane, L. P., Andres, P. G., Howland, K. C., Abbas, A. K. \& Weiss, A. Akt provides the CD28 costimulatory signal for up-regulation of IL-2 and IFN-gamma but not TH2 cytokines. Nature immunology 2, 37-44 (2001).

30. Fabian, M. A. et al. A small molecule-kinase interaction map for clinical kinase inhibitors. Nat Biotechnol 23, 329-336 (2005).

31. Lannutti, B. J. et al. CAL-101, a p110delta selective phosphatidylinositol-3-kinase inhibitor for the treatment of B-cell malignancies, inhibits PI3K signaling and cellular viability. Blood 117, 591-594 (2011).

32. Furman, R. R. et al. Idelalisib and rituximab in relapsed chronic lymphocytic leukemia. N Engl J Med 370, 997-1007 (2014).

33. Gopal, A. K. et al. PI3Kdelta inhibition by idelalisib in patients with relapsed indolent lymphoma. N Engl J Med 370, 1008-1018 (2014).

34. Su, D., Shen, M., Li, X. \& Sun, L. Roles of gammadelta T cells in the pathogenesis of autoimmune diseases. Clinical \& developmental immunology 2013, 985753 (2013).

35. Dubin, P. J. et al. Interleukin-23-mediated inflammation in Pseudomonas aeruginosa pulmonary infection. Infect Immun 80, 398-409 (2012)

36. Price, A. E., Reinhardt, R. L., Liang, H. E. \& Locksley, R. M. Marking and quantifying IL-17A-producing cells in vivo. PLoS One 7, e39750 (2012).

37. Wenzel, S. E. Asthma phenotypes: the evolution from clinical to molecular approaches. Nature medicine 18, 716-725 (2012).

38. Wehrens, E. J., Prakken, B. J. \& van Wijk, F. T cells out of control-impaired immune regulation in the inflamed joint. Nature reviews. Rheumatology 9, 34-42 (2013).

39. Sauer, S. et al. T cell receptor signaling controls Foxp3 expression via PI3K, Akt, and mTOR. Proceedings of the National Academy of Sciences of the United States of America 105, 7797-7802 (2008).

40. Merkenschlager, M. \& von Boehmer, H. PI3 kinase signalling blocks Foxp3 expression by sequestering Foxo factors. The Journal of experimental medicine 207, 1347-1350 (2010).

41. Alcorn, J. F., Crowe, C. R. \& Kolls, J. K. TH17 cells in asthma and COPD. Annu Rev Physiol 72, 495-516 (2010).

42. Bozic, C. R. et al. Expression and biologic characterization of the murine chemokine KC. Journal of immunology 154, 6048-6057 (1995).

43. Frevert, C. W., Huang, S., Danaee, H., Paulauskis, J. D. \& Kobzik, L. Functional characterization of the rat chemokine KC and its importance in neutrophil recruitment in a rat model of pulmonary inflammation. Journal of immunology 154, 335-344 (1995).

44. Semerad, C. L., Liu, F., Gregory, A. D., Stumpf, K. \& Link, D. C. G-CSF is an essential regulator of neutrophil trafficking from the bone marrow to the blood. Immunity 17, 413-423 (2002).

45. Akinleye, A., Avvaru, P., Furqan, M., Song, Y. \& Liu, D. Phosphatidylinositol 3-kinase (PI3K) inhibitors as cancer therapeutics. Journal of hematology \& oncology 6, 88 (2013).

46. Angulo, I. et al. Phosphoinositide 3-kinase delta gene mutation predisposes to respiratory infection and airway damage. Science 342, 866-871 (2013)

47. Lee, Y. K. et al. Late developmental plasticity in the T helper 17 lineage. Immunity 30, 92-107 (2009).

\section{Acknowledgements}

We thank Latesh Lad, David Koditek, and Annapurna Sapre of Gilead Sciences, Inc., for providing Compound B potency and selectivity data ahead of publication. This work was supported by NIH grants R37-HL079142 (KC and JKK) and T32-5T32AR052282-10 (EEW). EEW, KC, and JKK were supported by a research grant from Gilead Sciences, Inc., Seattle, WA. KC was also supported by the Research Advisory Committee of Children's Hospital of Pittsburgh of UPMC.

\section{Author Contributions}

E.E.W., J.K.K. and K.C. designed the experiment and wrote the manuscript, G.T.-N. participated in the in vivo experiment, L.P.K., B.H.S., K.D.P., J.K.K. and K.C. all participated in the design, acquisition of data, or analysis and interpretation of data, as well as manuscript writing.

\section{Additional Information}

Supplementary information accompanies this paper at http://www.nature.com/srep

Competing financial interests: Kamal Puri was Gilead employee and Bart Steiner is an employee of Gilead Sciences.

How to cite this article: Way, E. E. et al. Dose-Dependent Suppression of Cytokine production from T cells by a Novel Phosphoinositide 3-Kinase Delta Inhibitor. Sci. Rep. 6, 30384; doi: 10.1038/srep30384 (2016). 
(c) (i) This work is licensed under a Creative Commons Attribution 4.0 International License. The images or other third party material in this article are included in the article's Creative Commons license, unless indicated otherwise in the credit line; if the material is not included under the Creative Commons license, users will need to obtain permission from the license holder to reproduce the material. To view a copy of this license, visit http://creativecommons.org/licenses/by/4.0/

(C) The Author(s) 2016 